\title{
Self-Consistent Green Function Method in Nuclear Matter
}

\author{
Khaled S. A. Hassaneen ${ }^{1,2}$ \\ ${ }^{1}$ Department of Physics, Faculty of Science, Sohag University, Sohag, Egypt \\ ${ }^{2}$ Department of Physics, Faculty of Science, Taif University, Taif, Saudi Arabia \\ Correspondence should be addressed to Khaled S. A. Hassaneen; khs_94@yahoo.com
}

Received 28 April 2013; Accepted 20 June 2013

Academic Editor: Ali Hussain Reshak

Copyright (C) 2013 Khaled S. A. Hassaneen. This is an open access article distributed under the Creative Commons Attribution License, which permits unrestricted use, distribution, and reproduction in any medium, provided the original work is properly cited.

Symmetric nuclear matter is studied within the Brueckner-Hartree-Fock (BHF) approach and is extending to the self-consistent Green's function (SCGF) approach. Both approximations are based on realistic nucleon-nucleon interaction; that is, CD-Bonn potential is chosen. The single-particle energy and the equation of state (EOS) are studied. The Fermi energy at the saturation point fulfills the Hugenholtz-Van Hove theorem. In comparison to the BHF approach, the binding energy is reduced and the EOS is stiffer. Both the SCGF and BHF approaches do not reproduce the correct saturation point. A simple contact interaction should be added to SCGF and BHF approaches to reproduce the empirical saturation point.

\section{Introduction}

The correct treatment of short-range correlations when performing nuclear matter calculations using the basic nucleonnucleon interaction (NN) is of great importance [1]. One of these correlations has been studied using Brueckner-type resummation of ladder diagrams. This resummation allows to rewrite the ground-state energy of nuclear matter, using as an effective interaction the $G$-matrix, which takes care of the short-range repulsive core in the nucleon-nucleon interaction [2, 3]. Calculations using realistic interactions lead to results, which lie along a line (the Coester line) shifted with respect to the phenomenological saturation point $\left(\rho_{0} \approx\right.$ $\left.0.16 \mathrm{fm}^{-3}, E / A \approx-16 \mathrm{MeV}\right)[4]$. The remaining discrepancy can be attributed to relativistic effects and three-body forces contributions [5].

Self-consistent approaches based on the in-medium $T$ matrix approximation for nuclear matter have been studied [6]. In this way, a spectral function for nucleons in nuclear matter including two-particle correlations is obtained. The ladder diagrams involved in the calculation of the in-medium $T$-matrix include also hole-hole $(h h)$ propagation. The $T$ matrix approximation takes into account some of the higherorder hole line contributions as compared to the $G$-matrix approach. It would be instructive to study the saturation properties of nuclear matter for the self-consistent $G$-matrix approximation with realistic interactions.

Strictly speaking, however, the Bethe-Brandow-Petschek theorem [7] only defines the energy variable to be used in the calculation of self-energy or single-particle potential for the hole states. The choice for the propagator of the particle states is not defined on this level of the hole-line expansion and, therefore, has been discussed in a controversial way. The conventional choice has been to ignore self-energy contributions for the particle states completely and approximate the energies by the kinetic energy only. This conventional choice for the single-particle spectrum, however, is not very appealing as it leads to a gap at the Fermi surface; the propagator for single-particle states with momenta below the Fermi momentum $k_{F}$ is described in terms of a bound singleparticle energy while the corresponding spectrum for the particle states starts at the kinetic energy for the momentum $k_{F}$.

Jeukenne et al. [8] argued that it would be more natural to choose the propagator according to the Green function method, that is, define the single-particle propagator with a single-particle energy which includes the real part of the self-energy as a single-particle potential for particle and hole states. This leads to a spectrum that is continuous at the Fermi momentum, which provided the name "continuous 
choice" for this approach. This continuous choice leads to an enhancement of correlation effects in the medium and tends to predict larger binding energies for nuclear matter than the conventional choice.

In the present work, we want to explore some features of the single-particle energy and the corresponding Green function for infinite symmetric nuclear matter at zero temperature. As a starting point we consider the Brueckner-HartreeFoch (BHF) approximation employing a self-consistent continuous choice spectrum with an exact treatment of Pauli's operator $[9,10]$. The contribution of hole-hole ladder terms are then added in a perturbative approach [11]. Also, the binding energy of nuclear matter is studied.

\section{The Model}

2.1. BHF Approximation. Starting from realistic nucleonnucleon (NN) interaction, we have to use more advanced many-body approximations like the BHF which have the capability to account for the effects of correlations, which are due to the strong tensor and short-range components of such realistic NN interaction. The single-particle energy of a particle in the BHF approximation corresponds to the Hartree-Fock expression using the $G$-matrix for the effective interaction. This means that the self-energy of a nucleon in nuclear matter with momentum $k$ is given by (note that spin and isospin quantum numbers are suppressed) $[12,13]$

$$
\sum_{\mathrm{BHF}}(\vec{k}, \omega)=\int d^{3} q\langle\vec{k} \vec{q}|G(\Omega)| \vec{k} \vec{q}\rangle n_{0}(\vec{q}),
$$

with the occupation probability of a free Fermi gas with a Fermi momentum $k_{F}$ :

$$
n_{0}(\vec{q})= \begin{cases}1 & \text { for }|q| \leq k_{F} \\ 0 & \text { for }|q|>k_{F} .\end{cases}
$$

The matrix elements in (1) denote antisymmetrized matrix elements of the Brueckner $G$-matrix that are determined by solving the Bethe-Goldstone equation for a given realistic $\mathrm{NN}$ interaction $V$ :

$$
\begin{aligned}
& \langle\vec{k} \vec{q}|G(\Omega)| \vec{k} \vec{q}\rangle \\
& =\langle\vec{k} \vec{q}|V| \vec{k} \vec{q}\rangle+\int d^{3} p_{1} d^{3} p_{2}\left\langle\vec{k} \vec{q}|V| \overrightarrow{p_{1}} \overrightarrow{p_{2}}\right\rangle \\
& \quad \times \frac{Q\left(p_{1}, p_{2}\right)}{\Omega-\left(\varepsilon_{p_{1}}+\varepsilon_{p_{2}}\right)+i \eta}\left\langle\overrightarrow{p_{1}} \overrightarrow{p_{2}}|G(\Omega)| \vec{k} \vec{q}\right\rangle .
\end{aligned}
$$

The single-particle energies $\varepsilon_{p}$ of the intermediate states should be the corresponding BHF single-particle energies which are defined in terms of the real part of the BHF selfenergy of (1) by

$$
\varepsilon_{k}=\frac{k^{2}}{2 m}+\operatorname{Re}\left[\sum^{\text {BHF }}\left(k, \omega=\varepsilon_{k}\right)\right],
$$

with a starting energy parameter $\Omega=\omega+\varepsilon_{q}$ in the BetheGoldstone equation (3).
The Pauli operator $Q\left(p_{1}, p_{2}\right)$ restricts the intermediate states to particle states with momenta $p_{1}, p_{2}$, which are above the corresponding Fermi momentum. However, the singleparticle spectrum is often parameterized in the form of an effective mass

$$
\varepsilon_{k} \approx \frac{k^{2}}{2 m^{*}}+U
$$

so that a so-called angle-averaged propagator can be defined, which reduces the Bethe-Goldstone equation to an integral equation in one dimension. Hassaneen et al. [4] have studied the properties of nuclear matter in the framework of BHF approach with an angle-average approximation to the Pauli operator using five different two-body $\mathrm{NN}$ potentials. It is found that BHF theory is able to produce saturation due to short-range correlations but fails to reproduce the empirical region of saturation quantitatively $\left(\rho_{0}=0.16 \mathrm{fm}^{-3} ; E / A=\right.$ $-16 \mathrm{MeV})$. The exact Pauli operator has been treated in $[9,10]$.

2.2. Self-Consistent Green's Function. One of the drawbacks of the $\mathrm{BHF}$ approximation is the fact that it does not provide results for the equation of state, which are consistent from the point of view of thermodynamics. As an example we mention that $\mathrm{BHF}$ results do not fulfill, for example, the HugenholtzVan Hove theorem [14]. This is due to the fact that the BHF approximation does not consider the propagation of particle and hole states on equal footing. An extension of the BHF approximation, which obeys this symmetry, is the self-consistent Green's function (SCGF) method using the so-called $T$-matrix approximation. During the last years techniques have been developed, which allow to evaluate the solution of the SCGF equations for microscopic NN interactions [15-18]. Those calculations demonstrate that for the case of realistic NN interactions, the contribution of particleparticle ladders dominates the contribution of corresponding hole-hole propagation terms. This justifies the use of the BHF approximation and a procedure, which goes beyond $\mathrm{BHF}$ and accounts for hole-hole terms, in a perturbative way [11-13]. A perturbative treatment of hole-hole propagation avoids the appearance of the so-called pairing instabilities. A survey of pairing properties of nuclear matter media is presented in [19]. This leads to a modification of the self-energy in the BHF approximation by adding a hole-hole term of the form [11]

$$
\begin{aligned}
\Delta \sum^{2 h 1 p}(k, \omega)=\sum & \int_{k_{F}}^{\infty} d^{3} p \int_{0}^{k_{F}} d^{3} h_{1} \int_{0}^{k_{F}} d^{3} h_{2} \\
& \times \frac{\left\langle k p|G(\Omega)| h_{1} h_{2}\right\rangle^{2}}{\omega+\varepsilon_{p}-\varepsilon_{h_{1}}-\varepsilon_{h_{2}}+i \eta} .
\end{aligned}
$$

Using this expression of the self-energy induced by $h h$ contributions, we are not following the rules of holeline or Bethe-Brueckner-Goldstone expansion. In fact, the Bethe-Brandow-Petschek theorem [7] does not apply to the self-energy of hole states if this correction is used. More information about SCGF theory is reviewed by Dickhoff and Barbieri [20]. 
The quasiparticle energy for the extended self-energy can be defined as

$$
\varepsilon_{k}^{\mathrm{qp}}=\frac{k^{2}}{2 m}+\operatorname{Re}\left[\sum^{\mathrm{BHF}}\left(k, \omega=\varepsilon_{k}^{\mathrm{qp}}\right)+\Delta \sum^{2 h 1 p}\left(k, \omega=\varepsilon_{k}^{\mathrm{qp}}\right)\right] .
$$

The spectral functions for hole and particle strength, $S^{h}(k, \omega)$ and $S^{p}(k, \omega)$, are obtained from the real and imaginary parts of the self-energy $\sum=\sum^{\mathrm{BHF}}+\Delta \sum^{2 h 1 p}$

$$
\begin{aligned}
& S^{h(p)}(k, \omega) \\
& \quad= \pm \frac{1}{\pi} \frac{\operatorname{Im} \sum(k, \omega)}{\left[\omega-k^{2} / 2 m-\operatorname{Re} \sum(k, \omega)\right]^{2}+\left[\operatorname{Im} \sum(k, \omega)\right]^{2}},
\end{aligned}
$$

where the plus-minus sign on the left-hand side of this equation refers to the case of hole $\left(h, \omega<\varepsilon_{F}\right)$ and particle states $\left(p, \omega>\varepsilon_{F}\right)$, respectively.

In the case of two-body interactions, the hole spectral function gives access, through the Koltun sum rule [21], to the binding energy per particle:

$$
E / A(\rho)=\frac{\operatorname{deg}}{\rho(2 \pi)^{3}} \int d^{3} k \int_{-\infty}^{\varepsilon_{F}} \frac{1}{2}\left(\frac{k^{2}}{2 m}+\omega\right) S_{h}(k, \omega) d \omega,
$$

with deg denoting the degeneracy of the single-particle level, which is 4 for nuclear matter. The kinetic energy per nucleon is

$$
\begin{aligned}
t / A(\rho) & =\frac{\operatorname{deg}}{\rho(2 \pi)^{3}} \int d^{3} k \frac{k^{2}}{2 m} \int_{-\infty}^{\varepsilon_{F}} d \omega S_{h}(k, \omega) \\
& =\frac{\operatorname{deg}}{\rho(2 \pi)^{3}} \int d^{3} k \frac{k^{2}}{2 m} n(k),
\end{aligned}
$$

and the potential energy per particle has the form $v / A(\rho)=$ $E / A(\rho)-t / A(\rho)$. Introducing the removal energy $\varepsilon_{r}(k)$

$$
\varepsilon_{r}(k)=\frac{\int_{-\infty}^{\varepsilon_{F}} d \omega \omega S_{h}(k, \omega)}{\int_{-\infty}^{\varepsilon_{F}} d \omega S_{h}(k, \omega)},
$$

one can express the total binding energy per nucleon as

$$
E / A(\rho)=\frac{\operatorname{deg}}{\rho(2 \pi)^{3}} \int d^{3} k \frac{1}{2}\left(\frac{k^{2}}{2 m}+\varepsilon_{r}(k)\right) n(k) .
$$

In Brueckner-Hartree-Fock, $\varepsilon_{r}(k)=k^{2} / 2 m+u(k)$; that is, it coincides with the quasiparticle energy, and $n(k)$ denotes the step function, $n(k)=\theta\left(k_{F}-k\right)$; therefore,

$$
E / A^{\mathrm{BHF}}(\rho)=\frac{\operatorname{deg}}{\rho(2 \pi)^{3}} \int d^{3} k\left(\frac{k^{2}}{2 m}+\frac{1}{2} u(k)\right) .
$$

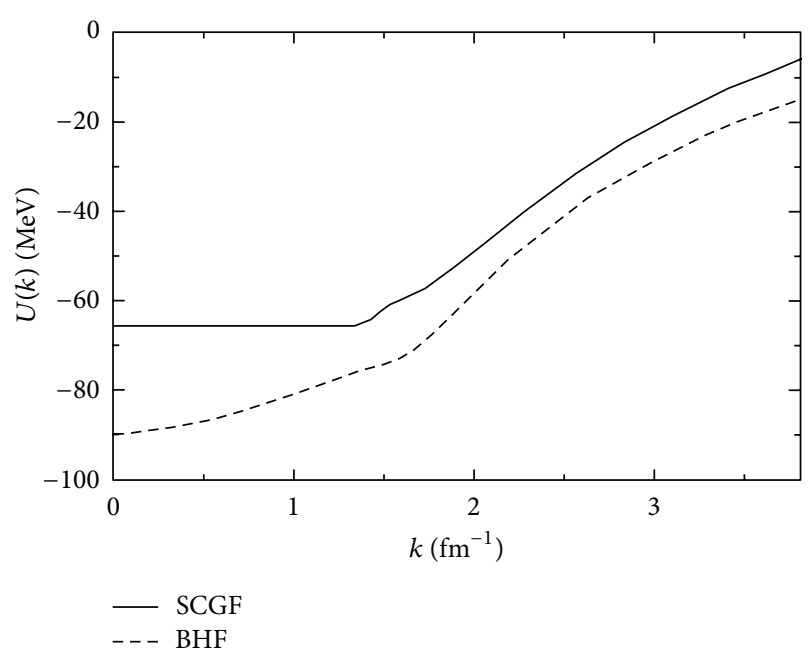

FIGURE 1: The single-particle potential or real part of self-energy as a function of momentum. The calculations are done for symmetric nuclear matter with a Fermi momentum $k_{F}=1.333 \mathrm{fm}^{-1}$ using CDBonn potential. The solid line represents the SCGF approximation, while the dashed one shows the BHF approach.

\section{Results and Discussion}

In Figures 1 and 2, we display the effect of hole-hole ladder contribution on the predicted self-energy or single-particle (s.p.) potential and single-particle energy or the quasiparticle energy $\left(\varepsilon_{\mathrm{qp}}\right)$. Results for the BHF approximation of s.p. potential and s.p. energy are shown with dotted line whereas the solid line represents the SCGF one. The calculations are done at the empirical value for the saturation density of symmetric nuclear matter $\left(\rho=0.16 \mathrm{fm}^{-3}\right)$ using CD-Bonn potential [22]. The corrections of the single-particle energies due to the $\Delta \sum^{2 h 1 p}$ term are larger for momenta below the Fermi momentum and tend to zero for momenta above $k_{F}$. This can be seen already from Figure 1 and is shown explicitly in Figure 2, where the single-particle potential, that is, the quasiparticle energy minus the kinetic energy, is compared to the corresponding value obtained in the BHF approximation. One finds that the single-particle potential derived from the quasiparticle energy tends to a constant for momenta below $k_{F}$. This means that the effective mass that describes the momentum dependence of the quasiparticle energy $\varepsilon_{\mathrm{qp}}$ is essentially equal to the bare mass for all momenta below $k_{F}$. At first sight, one may be tempted to consider this quasiparticle spectrum also in the energy denominators of the Bethe-Goldstone equation and the $2 h 1 p$ correction term of (6).

From Figure 1, one can see also that the BHF self-energy does not have a simple parabolic shape as a function of the momentum. There is a characteristic dip in the selfenergy always occurring at momenta slightly above the Fermi momentum of the kind of nucleons under consideration. The momentum dependence of the single-particle potential in a homogeneous infinite system is a sign of the nonlocality of the single-particle potential and is typically characterized in terms of an effective mass $m^{*}$, which can be used to 


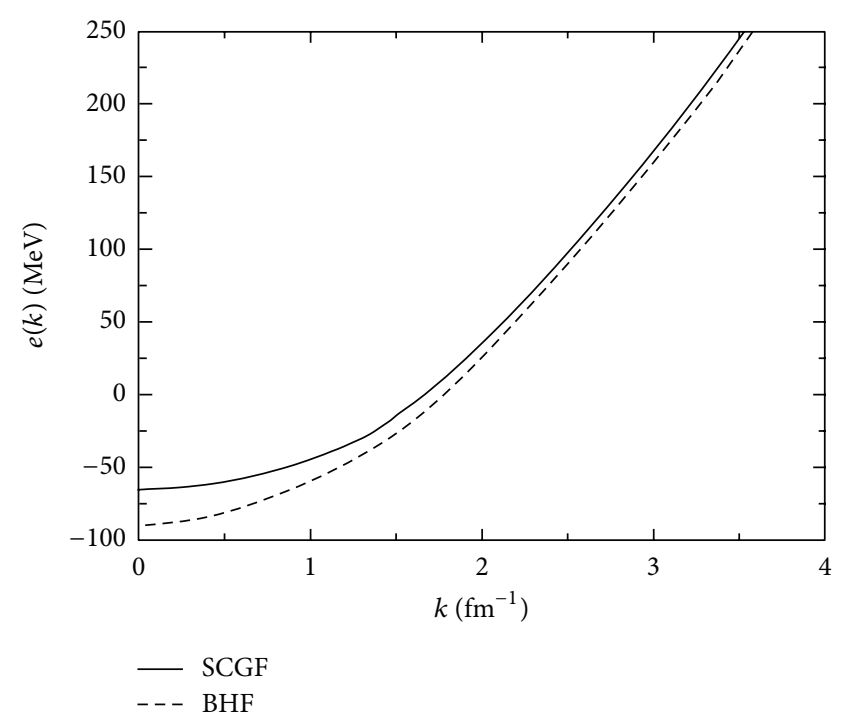

FIGURE 2: The single-particle energy determined from the SCGF approximation, solid line, and BHF approach, dashed line. The results are obtained for symmetric nuclear matter at a Fermi momentum $k_{F}=1.333 \mathrm{fm}^{-1}$ using CD-Bonn potential.

parameterize the momentum dependence of the singleparticle energies according to (5).

In Figure 3, the binding energy per particle of symmetric nuclear matter is plotted versus the density. The results of the full SCGF calculation, computed at a temperature of $T=0 \mathrm{MeV}$, are indicated by solid line passing through solid circles in Figure 3. The BHF results are indicated by the triangles that are connected by a solid line. The empirical saturation point is given by the big square. The hole-hole contributions to the nucleon self-energy, which are included in the SCGF approach, yield a repulsive contribution the energy per nucleon, which increases with increasing nuclear density. This shifts the saturation point to a lower density and binding energy per nucleon so that also the saturation point obtained with SCGF is within the Coester band. Due to this effect, the saturation density in the SCGF approximation, $\rho_{\text {sat }}=0.211 \mathrm{fm}^{-3}$ and $E_{\text {sat }}=-11.34 \mathrm{MeV}$, is considerably smaller than the saturation density in the BHF approach that has the value $\rho_{\text {sat }}=0.391 \mathrm{fm}^{-3}$ and $E_{\text {sat }}=-23.97 \mathrm{MeV}$. After all the saturation density is reduced by almost $50 \%$; however, both values are not near the empirical one $\left(\rho_{\text {sat }} \approx 0.16 \mathrm{fm}^{-3}\right.$, $E_{\text {sat }} \approx-16 \mathrm{MeV}$ ).

A nice property of the SCGF approach is its thermodynamic consistency. In particular, it fulfills the HugenholtzVan Hove theorem, which states that whenever a further nucleon is added to the nuclear system in equilibrium, that is, at the saturation point, the energy gain is exactly the binding energy of a nucleon [14], the chemical potential $\mu=E_{\text {sat }}$. In Figure 3, the chemical potentials are given by the dotted lines. The violation of the theorem in the zero temperature continuous choice BHF calculation amounts to about $20 \mathrm{MeV}$.

In order to obtain the experimental saturation value in a symmetric nuclear matter, one has to add threebody interaction terms or a density-dependent two-nucleon
TABLE 1: The values of the parameters $t_{0}$ and $t_{3}$ that are used to fit the saturation point.

\begin{tabular}{lcc}
\hline Parameters & BHF & SCGF \\
\hline$t_{0}$ & -221.5 & -326.7 \\
$t_{3}$ & 3749.9 & 3916.9 \\
\hline
\end{tabular}

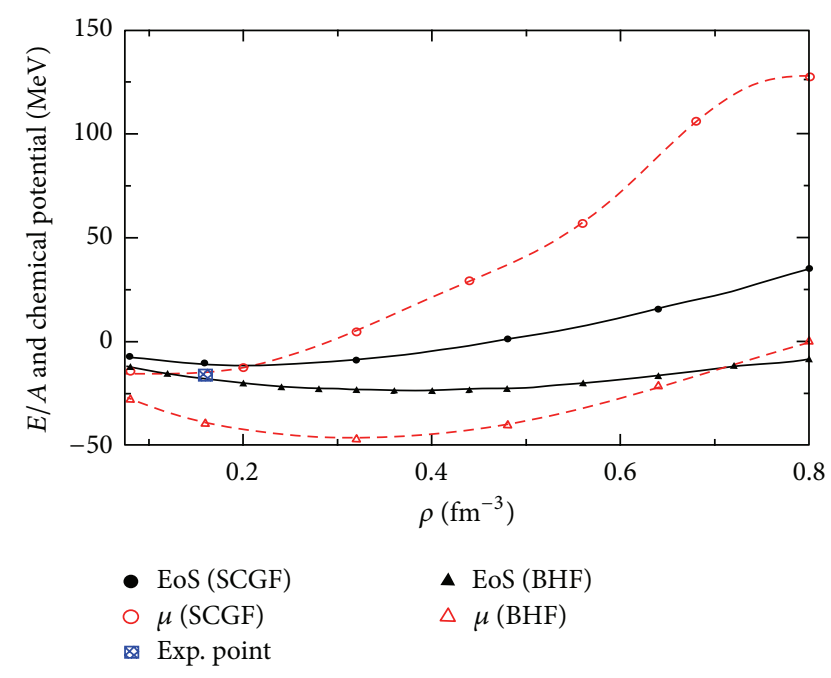

FIgure 3: The binding energy per nucleon $E / A$ (black curves) and the chemical potential $\mu$ (red curves) as a function of the density $\rho$. The box indicates the empirical saturation point. See text for details.

interaction. Therefore, it is quite natural to supplement the effective interaction or the self-energy of BHF and SCGF calculations by a simple contact interaction, which we have chosen following the notation of the Skyrme interaction to be of the form

$$
E^{\mathrm{corr}} / A=\frac{1}{2} t_{0} \rho+\frac{1}{12} t_{3} \rho^{1+\alpha},
$$

where $\rho$ is the matter density and $t_{0}, t_{3}$, and $\alpha$ are parameters. For a fixed value of $\alpha$ (typically $\alpha=0.5$ ), we have fitted $t_{0}$ and $t_{3}$ in such a way that BHF and SCGF calculations plus the contact term (ct) of (14) yield the empirical saturation point for symmetric nuclear matter; that is, $E^{\text {corr }} / A=$ ct. The results for these fitting parameters are listed in Table 1, and the corresponding energy versus density curves are displayed in Figure 4. For all cases, the fit yields an attractive two-body contact interaction and a repulsive $t_{3}$ term. The results are compared to those obtained by Baldo and Shaban [23] and supplemented by a microscopic three-body force and relativistic BHF. One can see from Figure 4, that the present calculations plus the contact term give stiffer EOS like relativistic BHF especially at high density [24].

\section{Conclusion}

The single-particle energy is calculated self-consistently using BHF and SCGF approximations. The contribution of the $h h$ terms leads to a repulsive contribution to the single-particle energy which decreases with momentum. This implies that 


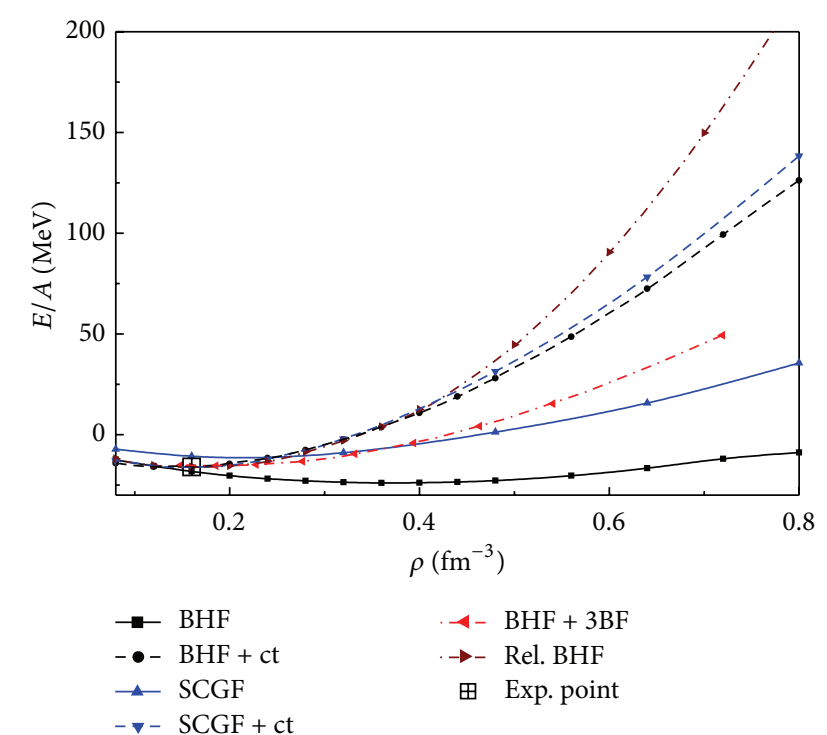

FIgURE 4: $E / A$ in $\mathrm{MeV}$ for symmetric nuclear matter as a function of density using CD-Bonn potential. The results are obtained using both BHF and SCGF approaches with and without an additional contact interaction of the form displayed in (14), in comparison with microscopic three-body force by Baldo and Shaban [23] and relativistic BHF [24] by Müther et al.

the equation of state derived within the SCGF approach tends to be stiffer than the corresponding one evaluated within the $\mathrm{BHF}$ approximation. Also, the inclusion of $h h$ propagation in the SCGF approach leads to a somewhat stiffer chemical potential than in BHF approximation. The Hugenholtz-Van Hove theorem is well fulfilled in the SCGF approximation while it is badly violated in the BHF approach.

Therefore, the present approximation should avoid the so-called pairing instability which occurs in the SCGF approach using the Galitskii-Feynman propagator. A more systematic study of symmetric nuclear should be useful, which goes beyond the present model and treats the propagation of hole-hole scattering terms in a nonperturbative way $[15-18,25]$.

Both of the present methods fail to reproduce the empirical saturation point. Thus, these microscopic approaches are supplemented by a density-dependent contact term to obtain the empirical saturation point of symmetric nuclear matter.

\section{References}

[1] H. Müther and A. Polls, "Two-body correlations in nuclear systems," Progress in Particle and Nuclear Physics, vol. 45, no. 1, pp. 243-334, 2000.

[2] K. A. Brueckner and J. L. Gammel, "Properties of nuclear matter," Physical Review, vol. 109, no. 4, pp. 1023-1039, 1958.

[3] B. D. Day, "Elements of the Brueckner-Goldstone theory of nuclear matter," Reviews of Modern Physics, vol. 39, no. 4, pp. 719-744, 1967.

[4] K. S. A. Hassaneen, H. M. Abo-Elsebaa, E. A. Sultan, and H. M. M. Mansour, "Nuclear binding energy and symmetry energy of nuclear matter with modern nucleon-nucleon potentials,' Annals of Physics, vol. 326, no. 3, pp. 566-577, 2011.

[5] R. Brockmann and R. Machleidt, "Relativistic nuclear structure. I. Nuclear matter," Physical Review C, vol. 42, no. 5, pp. 19651980.

[6] T. Frick, K. S. A. Hassaneen, D. Rohe, and H. Müther, "Spectral function at high missing energies and momenta," Physical Review C, vol. 70, no. 2, Article ID 024309, 5 pages, 2004.

[7] H. A. Bethe, B. H. Brandow, and A. G. Petschek, "Reference spectrum method for nuclear matter," Physical Review, vol. 129, no. 1, pp. 225-264, 1963.

[8] J. P. Jeukenne, A. Lejeune, and C. Mahaux, "Many-body theory of nuclear matter," Physics Reports, vol. 25, no. 2, pp. 83-174, 1976.

[9] E. Schiller, H. Müther, and P. Czerski, "Pauli exclusion operator and binding energy of nuclear matter," Physical Review C, vol. 59, no. 5, pp. 2934-2936, 1999.

[10] E. Schiller, H. Müther, and P. Czerski, "Erratum: pauli exclusion operator and binding energy of nuclear matter [Physical Review C, 59, 2934 (1999)]," Physical Review C, vol. 60, no. 5, Article ID 059901(E), 1 page, 1999.

[11] P. Grangé, J. Cugnon, and A. Lejeune, "Nuclear mean field with correlations at finite temperature," Nuclear Physics A, vol. 473, no. 3, pp. 365-393, 1987.

[12] T. Frick, Kh. Gad, H. Müther, and P. Czerski, "Nuclear selfenergy and realistic interactions," Physical Review C, vol. 65, no. 3, Article ID 034321, 13 pages, 2002.

[13] K. S. A. Hassaneen and H. Müther, "Correlations and spectral functions in asymmetric nuclear matter," Physical Review C, vol. 70, no. 5, Article ID 054308, 8 pages, 2004.

[14] N. M. Hugenholz and L. van Hove, "A theorem on the single particle energy in a Fermi gas with interaction," Physica, vol. 24, no. 1-5, pp. 363-376, 1958.

[15] Y. Dewulf, W. H. Dickhoff, D. van Neck, E. R. Stoddard, and M. Waroquier, "Saturation of nuclear matter and short-range correlations," Physical Review Letters, vol. 90, no. 15, Article ID 152501, 4 pages, 2003.

[16] P. Bożek, "One-body properties of nuclear matter with off-shell propagation," Physical Review C, vol. 65, Article ID 054306, 10 pages, 2002.

[17] T. Frick and H. Müther, "Self-consistent solution to the nuclearmany-body problem at finite temperature," Physical Review C, vol. 68, no. 3, Article ID 034310, 9 pages, 2003.

[18] T. Frick, H. Müther, A. Rios, A. Polls, and A. Ramos, "Correlations in hot asymmetric nuclear matter," Physical Review C, vol. 71, no. 1, Article ID 014313, 8 pages, 2005.

[19] H. Müther and W. H. Dickhoff, "Pairing properties of nucleonic matter employing dressed nucleons," Physical Review C, vol. 72, no. 5, Article ID 054313, 11 pages, 2005.

[20] W. H. Dickhoff and C. Barbieri, "Self-consistent Green's function method for nuclei and nuclear matter," Progress in Particle and Nuclear Physics, vol. 52, no. 2, pp. 377-496, 2004.

[21] D. S. Koltun, "Theory of mean removal energies for single particles in nuclei," Physical Review C, vol. 9, no. 2, pp. 484-497, 1974.

[22] R. Machleidt, "High-precision, charge-dependent Bonn nucleon-nucleon potential," Physical Review C, vol. 63, no. 2, Article ID 024001, 32 pages, 2001.

[23] M. Baldo and A. E. Shaban, "Dependence of the nuclear equation of state on two-body and three-body forces," Physics Letters B, vol. 661, no. 6, pp. 373-377, 2008. 
[24] H. Müther, M. Prakash, and T. L. Ainsworth, "The nuclear symmetry energy in relativistic Brueckner-Hartree-Fock calculations," Physics Letters B, vol. 199, no. 4, pp. 469-474, 1987.

[25] A. Rios, A. Polls, and I. Vidaña, "Hot neutron matter from a selfconsistent Green's-functions approach," Physical Review C, vol. 79, no. 2, Article ID 025802, 13 pages, 2009. 

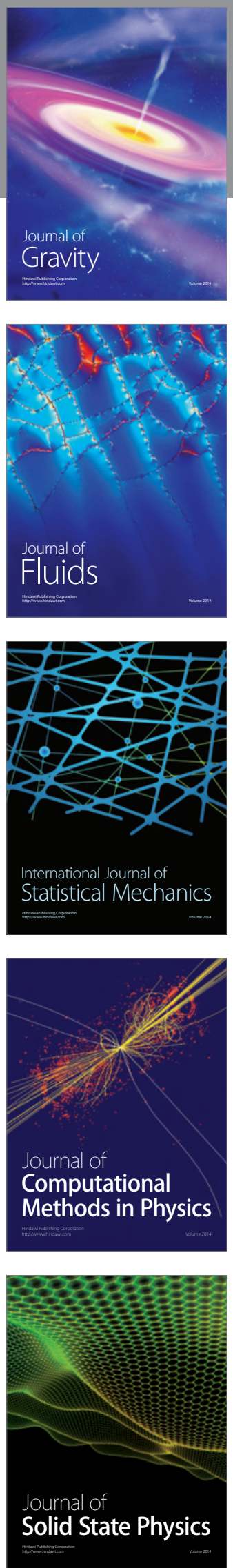

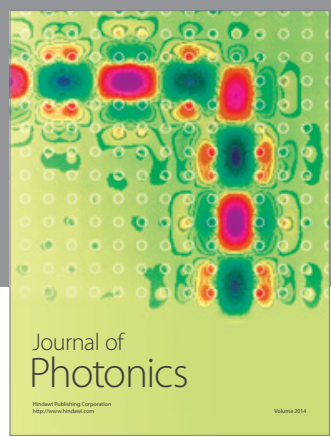

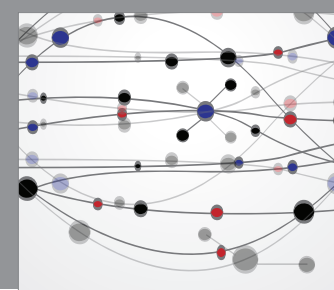

The Scientific World Journal

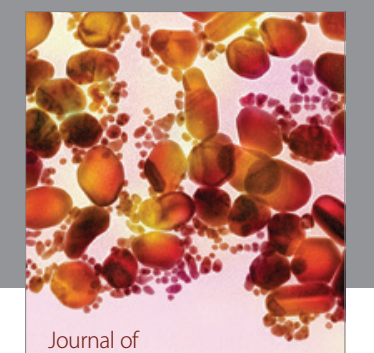

Soft Matter
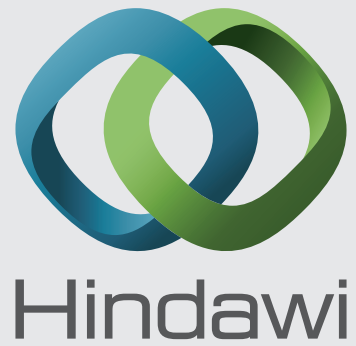

Submit your manuscripts at

http://www.hindawi.com
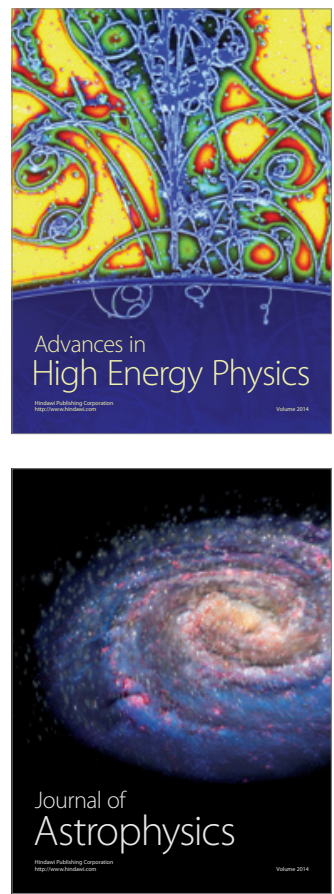
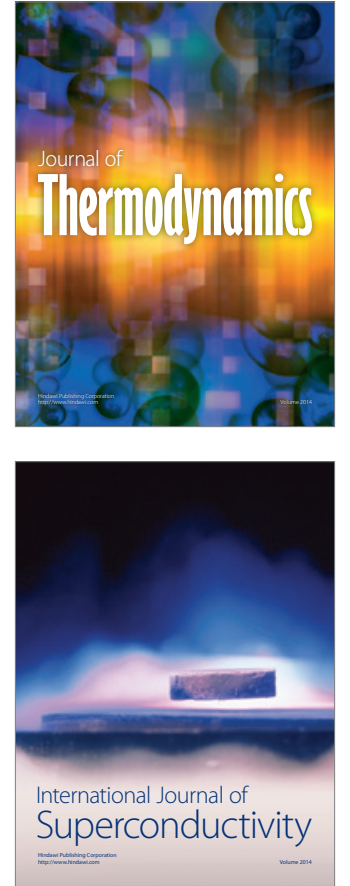
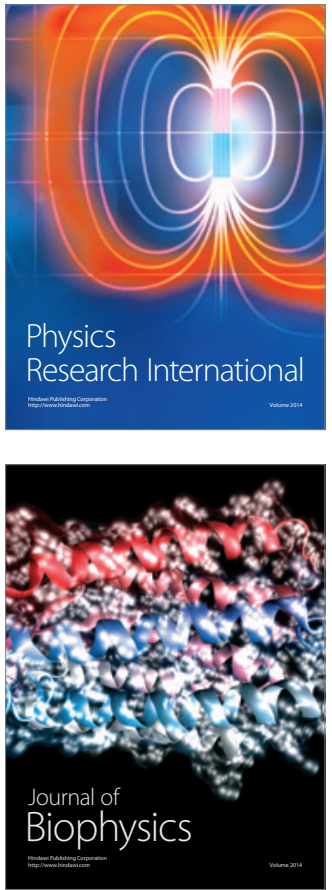
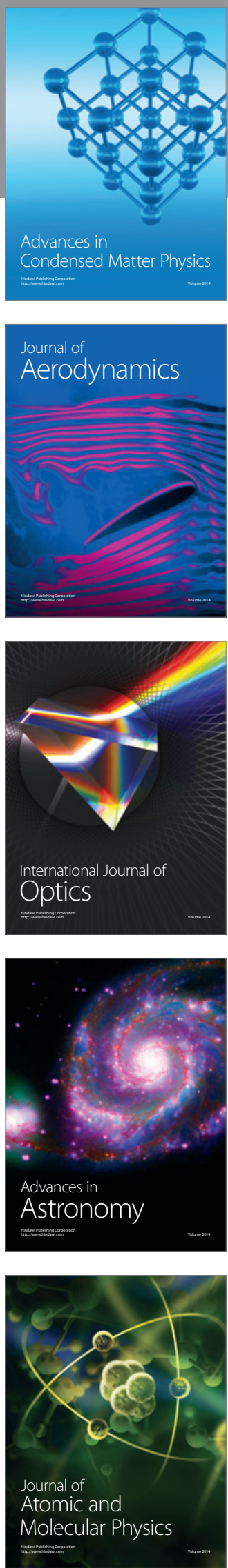